\title{
Formation OF EQUivalent MODELS OF STRUCTURED MULTIRATE SySTEMS IN THE FORM OF SigNAL GRAPH
}

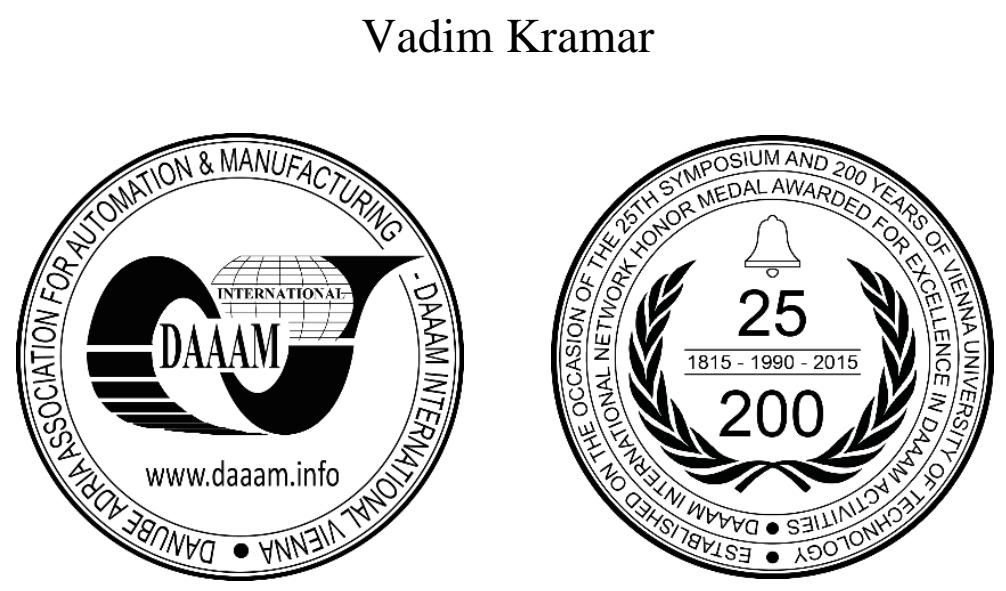

This Publication has to be referred as: Kramar, V[adim] (2017). Formation of Equivalent Models of Structured Multirate Systems in the Form of Signal Graph, Proceedings of the 28th DAAAM International Symposium, pp.00870092, B. Katalinic (Ed.), Published by DAAAM International, ISBN 978-3-902734-11-2, ISSN 1726-9679, Vienna, Austria

DOI: $10.2507 / 28$ th.daaam.proceedings.011

\begin{abstract}
The usage of microprocessors in the measuring and processing channels along with continuously working arrangement is typical for modern control systems. Similar control systems carry out measurements and processing of some signals sharing time. Mathematical models of such systems are represented in a multivariate multistep discrete control systems. Investigations of similar systems lead to the need of their model development in a complex range, with transfer functions as main modelling elements. Signal graph provides evident representation of system variables and their interaction. This article gives a way to the creation of a signal graph one-time uninterruptedly discrete system for which the Mason equation can be applied. Thus, the problem of process analysis in given multirate systems becomes reduced to the problem of rational functions.
\end{abstract}

Keywords: Multidimensional systems; digital-analogue systems; signal graph; Mason equation; equivalent model

\section{Introduction}

The usage of microprocessors in the measuring and processing channels along with continuously working arrangement is typical for modern automatic control systems [1-4]. As a rule, similar control systems carry out measurements and processing of some signals sharing time. Mathematical models of such systems are represented in a multivariate multistep uninterruptedly discrete automatic control systems. Investigations of similar systems lead to the need of their model development in a complex range, with transfer functions as main modelling elements. To obtain control systems models in the form of transfer functions, methods using signal graph have found a wide applicationx[5,6]. Signal graph provides evident representation of system variables and their interaction. It is well known that to determine transfer functions of linear permanent systems the Mason equation can be applied. In [7] approaches of Mason equation [8] application in onetime uninterruptedly discrete systems are observed. If there are some various times, the Coffi and Williams matrix approach should be applied. This article gives a way to the creation of a signal graph one-time uninterruptedly discrete system for which the Manson equation can be applied.

While constructing signal graph of multirate uninterrupted discrete system, let us consider the symbol system of Sedler and Becky $[9,10]$. White knot in the graph is used to define uninterrupted variable system. Black knot is used to define discrete variable and quantum operations, variable significance represented by any black knot being discrete form of sum transformation of all variables, being in the knot, according to a certain rate. Because in uninterrupted discrete, as a rule, 
it is impossible to outline inlet variable in presentation for, thus it is worthwhile to coordinate inlet influence. It is carried out by introduction of branches with transfer function equal to inlet variables so that entire transfer function belonging to the single output, transfer function and output become equal.

\section{Formation of equivalent mathematic model of structured multirate systems}

Linear uninterrupted discrete system with some quantifiers with discrete time $T_{1}, \ldots, \mathrm{T}_{\mathrm{N}}$ can be described by the system of linear algebraic equations in certain fields

$$
A(s) x(s)=\sum_{i=1}^{N} B_{i} x^{T i}(s)+R
$$

where: $x(s)-n$ is vector of variable systems represented according to Laplace; $A(s)$ - $n \times n$ uninterrupted transfer function matrix; $x^{T i}(s)$ - discrete transformation in times $T_{i}, i=1, \ldots, N$ vector $x(s) ; R-n$-standardized input vector which, according to system linearity, can be considered as vector $R^{\prime}=\left[\begin{array}{lllll}1 & 0 & 0 & \ldots & 0\end{array}\right]^{\prime}$, where ' - here and further means transportation; $B_{i}-n \times n-$ matrixes characterizing quantifier presence with various discrete times in system, they consist of 0 and 1 and represent quantification.

To simplify (1) it is worthwhile to write first of all equations for uninterrupted, and then for quantum variables by turns for each discrete time $T_{i}, i=1, \ldots, N[1] . A(s)$ is of block type

$$
A(s)=\left[\begin{array}{cccc}
W(s) & \Phi_{1}(s) & \cdots & \Phi_{N}(s) \\
0 & I_{1} & \cdots & 0 \\
\vdots & \vdots & \ddots & \vdots \\
0 & 0 & \cdots & I_{N}
\end{array}\right]
$$

where: $N$ - quantity of different measure point; $W(s)$ - $l \times l$ - transfer function matrix; $l$ - uninterrupted variables number; $\Phi_{i}(s)-l \times m_{i}-$ transfer function matrix; $m_{i}$ - quantifier number with discrete time $T_{i}, i=1, \ldots, N ; I_{i}$ - identity matrixes $m_{i}$ $\times m_{i}$.

Let us define

$$
y_{i}^{T_{i}}(s)=B_{i} x^{T_{i}}(s), i=1, \ldots, N
$$

Vector $y_{i}^{T_{i}}(s)$ consists of the $m_{i}$ vector component $x^{T_{i}}$, complying with quantifier outputs with discrete time $T_{i}$. From (1), taking into account (3) (considering the presence of $A^{-1}(s)$ ), we obtain

$$
x(s)=A^{-1}(s) \sum_{j=1}^{N} y_{j}^{T_{j}}(s)+A^{-1}(s) R
$$

Elements $a_{i j}^{-1}$ of matrix $A^{-1}$ are transfer functions from $j$ knot to $i$ knot of initial system with all open quantifiers. Actually, considering quantifiers and vectors $y_{i}^{T_{i}}(s)$ to be independent input effects which can be defined as zero, from (4) we obtain

$$
x_{i}(s)=\sum_{j=1}^{N} a_{i j}^{-1} r_{j}
$$

where: $r_{j}$ - vector elements of normalized input $R$.

On the basis of (4), uninterrupted discrete system 'complex graph' can be created, which gives us system transfer functions by applying the Manson formula. A complex graph is uninterrupted discrete system initial graph and discrete graph combination, made up $y_{i}^{T_{i}}(s)$. In (4), variables $y_{i}^{T_{i}}(s)$ are input signals of keys which we will define as input variables. Having formed $N$ discrete graph for $y_{i}^{T_{i}}(s)$, we may delete quantifiers from the initial graph and connect input knots of keys with equivalent knots of discrete graphs by means of branches with identity intensification coefficients. In this way, 
we obtain a complex system graph to which the Manson formula is applicable. Consider uninterrupted discrete system with $\mathrm{N}$ quantizers, so that discreteness es are as follows

$$
\frac{T_{i}}{T_{i+1}}=\frac{b_{i}}{q_{i}} \quad i=1, \ldots, N-1
$$

The system of linear algebraic equations with the time rate for this system is shown in (4). Multiplying (4) to the correspondent $B_{i}$, we obtain a ratio system

$$
y_{i}(s)=B_{i} A^{-1}(s) \sum_{j=1}^{N} y_{j}^{T_{j}}+B_{i} A^{-1}(s) R \quad i=1, \ldots, N
$$

Let $T$ - discrete time - be equal to the least common multiple time of all $N$ times $T_{i}$.

We can carry out discrete conversion of vector $x(s)$ to least common multiple - time $T$

$$
x^{T}(s)=\left(A^{-1}(s) \sum_{j=1}^{N} y_{j}^{T_{j}}(s)\right)^{T}+\left(A^{-1}(s) R\right)^{T}
$$

Assuming the meaning of $\frac{T}{T_{i}}=n_{i}$, and using discrete transformer property

$$
\begin{aligned}
{\left[g(s) z^{T}(s)\right]^{n T}=} & \sum_{i=o}^{n-1}\left(g(s) e^{-i T s}\right)^{n T}\left(z(s) e^{i T s}\right)^{n T}, \text { we can write } \\
x^{T}(s)= & \sum_{i_{1}=0}^{n_{1}-1}\left(A^{-1}(s) e^{-i_{1} T_{1} s}\right)^{T}\left(y_{1}(s) e^{i_{1} T_{1} s}\right)^{T}+\ldots+ \\
& +\sum_{i_{N}=0}^{n_{N}-1}\left(A^{-1}(s) e^{-i_{N} T_{N} s}\right)^{T}\left(y_{N}(s) e^{i_{N} T_{N} s}\right)^{T}+ \\
& +\left(A^{-1}(s) R\right)^{T}
\end{aligned}
$$

Let us identify

$$
\begin{aligned}
& Y_{1\left(i_{1}\right)}=y_{1}(s) e^{i_{1} T_{1} s} \ldots Y_{N\left(i_{N}\right)}=y_{N}(s) e^{i_{N} T_{N} s} \\
& i_{j}=0, \ldots,\left(n_{j}-1\right) ; \quad j=1, \ldots, N
\end{aligned}
$$

We obtain equation of vectors $Y_{1(i)}^{T}, \ldots, Y_{N(i)}^{T}$, then multiply in (8) each equation to $e^{i T_{i} s}\left(i_{j}=0, \ldots,\left(n_{j}-1\right), \quad j=1, \ldots, N\right)$.

$$
\left\{\begin{array}{c}
Y_{1\left(i_{1}\right)}=\left(B_{1} A^{-1}(s) e^{i_{1} T_{1} s} \sum_{j=1}^{N} y_{j}^{T_{j}}(s)\right)+\left(B_{1} A^{-1}(s) \operatorname{Re}^{i_{1} T_{1} s}\right) \\
i_{1}=0, \ldots,\left(n_{1}-1\right) \quad \begin{array}{c}
\vdots \\
Y_{N\left(i_{N}\right)}=\left(B_{N} A^{-1}(s) e^{i_{N} T_{N} s} \sum_{j=1}^{N} y_{j}^{T_{j}}(s)\right)+\left(B_{N} A^{-1}(s) \operatorname{Re}^{i_{N} T_{N} s}\right) \\
i_{N}=0, \ldots,\left(n_{N}-1\right)
\end{array}
\end{array}\right.
$$

Let us carry out discrete transformation (10) to the least common multiple - time $T$ 


$$
\left\{\begin{aligned}
Y_{1\left(i_{1}\right)}^{T}= & \sum_{k_{1}=0}^{n_{1}-1}\left(\bar{W}_{1\left(i_{1}\right)} e^{-k_{1} T_{1} s}\right)^{T} Y_{1\left(k_{1}\right)}^{T}+ \\
& +\sum_{k_{2}=0}^{n_{2}-1}\left(\bar{W}_{1\left(i_{1}\right)} e^{-k_{2} T_{2} s}\right)^{T} Y_{2\left(k_{2}\right)}^{T}+\ldots+ \\
& +\sum_{k_{N}=0}^{n_{N}-1}\left(\bar{W}_{1\left(i_{1}\right)} e^{-k_{N} T_{N} s}\right)^{T} Y_{N\left(k_{N}\right)}^{T}+\Phi_{(1)}^{T} \\
i_{1}= & 0, \ldots,\left(n_{1}-1\right) \\
Y_{N\left(i_{N}\right)}^{T} & \sum_{k_{1}=0}^{n_{1}-1}\left(\bar{W}_{N\left(i_{N}\right)} e^{-k_{1} T_{1} s}\right)^{T} Y_{1\left(k_{1}\right)}^{T}+ \\
& +\sum_{k_{2}=0}^{n_{2}-1}\left(\bar{W}_{N\left(i_{N}\right)} e^{-k_{2} T_{2} s}\right)^{T} Y_{2\left(k_{2}\right)}^{T}+\ldots+ \\
& +\sum_{k_{N}=0}^{n_{N}-1}\left(\bar{W}_{N\left(i_{N}\right)} e^{-k_{N} T_{N} s}\right)^{T} Y_{N\left(k_{N}\right)}^{T}+\Phi_{(N)}^{T} \\
i_{N}= & 0, \ldots,\left(n_{N}-1\right)
\end{aligned}\right.
$$

where:

$$
\begin{array}{ll}
\bar{W}_{j\left(i_{j}\right)}=B_{j} A^{-1}(\mathrm{~s}) e^{i_{j} T_{j} s} & j=1, \ldots, N, \\
\Phi_{j}=B_{j} A^{-1}(s) R e^{i_{j} T_{j} s} & j=1, \ldots, N .
\end{array}
$$

Calculating together (12) and (10) and introducing $Y_{i}$ we may obtain input-output relation for all variable systems. This system contains

$$
n+\sum_{j=1}^{N} m_{j} n_{j}
$$

equation, where: $\mathrm{n}$ - vector dimension $x(s) ; m_{j}$ - number of keys with times $T_{j} ; n_{j}$ - number equal to the relation of the least common multiple time to time $T_{j}$. Ratio (10), after introducing $Y_{i}$, can be accepted as discrete system description, inputs of which along with outputs of initial uninterruptedly discrete system are variables $Y_{j\left(i_{j}\right)}^{T} \quad j=1, \ldots, N ; \quad i_{j}=0, \ldots,\left(n_{j}-1\right)$. As their representation is

$$
y_{j}^{T_{j}}=\sum_{i_{j}=0}^{n_{j}-1} e^{-i_{j} T_{j} s}\left[y_{j} e^{i_{j} T_{j} s}\right]^{T}=\sum_{i_{j}=0}^{n_{j}-1} e^{-i_{j} T_{j} s} Y_{j\left(i_{j}\right)}^{T}
$$

it can be considered to be a result of input signals parallelization of keys with time $T_{j}$ in the initial system on $\mathrm{n}_{\mathrm{j}}$ branches, each of which has transfer coefficient

$$
e^{-i_{j} T_{j} s} \quad i_{j}=0, \ldots,\left(n_{j}-1\right)
$$

Then, the initial system in (9) can be specified by the discrete graph in which keys are open and their outputs are input signals into the system along with initial input signals. At the same time, each of these new inputs from quantifiers $T_{j}($ $j=1, \ldots, N)$ can be represented as a set of inputs $Y_{j\left(i_{j}\right)}^{T} j=1, \ldots, N ; i_{j}=0, \ldots,\left(n_{j}-1\right)$ and, by connecting certain knots by identity connections, we can formulate the input-output ratio according to the final discrete graph. 
Discrete graphs for $Y_{j\left(i_{j}\right)}^{T}$ are built on the basis of (12). There should be $k=\sum_{j=1}^{N} n_{j}$ discrete graphs.

\section{Algorithm for uninterruptedly discrete system signal graph}

The construction of algorithm for uninterruptedly discrete system signal graph on the basis of equations (10) - (12) will be as follows:

1. On the basis of structure scheme an uninterruptedly discrete system initial graph is formed. All quantifiers in it, $T_{j} j=$ $1, \ldots, N$, are considered to be regulated by diminution open, at the same time output signals of keys are considered to be input ones into the system where $\sum_{j=1}^{N} m_{j}$ is the quantity of black knots and $\mathrm{m}_{\mathrm{j}}-$ key number with time $T_{j}$.

2. Form $n_{j} \quad j=1, \ldots, N$ of the discrete graph, corresponding to time $T_{j}$ on the following procedures: a1) $j=1$;

a) in the initial graph we compose only knots corresponding to quantifiers input signals with time $T_{j}$ and connected with them input knots (initial and from quantifiers). As a result we obtain the intermediate graph;

a2) $i_{j}=0$;

b) in the intermediate graph we replace all transfer functions of links $W$ with $W e^{i_{j} T_{j} s}$;

c) we make input signals parallel from keys $T_{k}, k=1, \ldots, N, k \neq j$ to $\mathrm{n}_{\mathrm{k}}$ branches, substituting each transfer function of the branch $W$ for $W e^{-l_{k} T_{k} s} \quad l_{k}=0, \ldots,\left(n_{k}-1\right), k=1, \ldots N, k \neq j$.

d) we substitute knots for black, and transfer functions for their discrete transformation in time T. We enter input knots in the Table through $Y_{k\left(i_{k}\right)}^{T}$, then state conformity between knots $x^{T}$ и $Y_{k\left(i_{k}\right)}^{T}$ by means of identity connections, where it is necessary. We obtain a discrete graph corresponding to time $T_{j}$.

e) points $\mathrm{b}) \div \mathrm{d}$ ) are now executed $\mathrm{n}_{\mathrm{j}}$ times, supposing $i_{j}=i_{j}+1$; this results in discrete graph $\mathrm{n}_{\mathrm{j}}$, corresponding to time $T_{j}, i_{j}=0, \ldots,\left(n_{j}-1\right)$;

f) $j$ - we increase by one and repeat points a) $\div$ e) for discrete graph construction for all $\mathrm{N}$ quantifiers, i.e. $j=1, \ldots, N$.

3. Now we turn back to the initial graph and make all input signals parallel from keys $y_{j}^{T_{j}}, j=1, \ldots, N$, into $\mathrm{n}_{\mathrm{j}}$ corresponding branches $j=1, \ldots, N$.

We transfer each function from $\mathrm{n}_{\mathrm{j}}$ branches $W$ and substitute for $W e^{-k_{j} T_{j} s} \quad k_{j}=0, \ldots,\left(n_{j}-1\right), j=1, \ldots N$, respectively.

We now enter the input knots in the Table through variables $Y_{j\left(i_{j}\right)}^{T}, i_{j}=0, \ldots,\left(n_{j}-1\right)$.

We connect to the marked inputs all of discrete graphs, got in item 2, by single connections. We set, where necessary, other single connections, proper identical knots. We get the component graph.

4. We substitute all the knots with black, and transfer functions on their discrete transformations on time the least common multiple - on time of $\mathrm{T}$. We get the final discrete graph of the system.

5. By applying the Mason rule we determined the necessity of input-output correlation for the system variables of $x^{T}$ for the obtained graph of the system.

Now we can formulate the algorithm of receipt of signal graph of multivariate of the continuously-discrete system for the case of multiple times.

1. On the basis of the flow diagram of the system, an initial graph containing white and black knots is formed. Black knots have indexes of $T i, I=1, . ., N$, proper to the value of time of discreteness. Times are considered well-organized on a decrease. In the initial graph keys are considered broken; here the output signals of keys are considered entrance knots in the system and black knots correspond to them.

2. On the initial graph of the system, through the application of the Mason algorithm, the discrete graph of $1 \mathrm{st}$ level is formed in conformance with the following: 
All the knots, being by an entrance for the keys with the smallest time of $T N$, get out in the initial graph. They are considered output knots. All the entrance knots, related to the indicated output, get out then. Entrance knots can be the entrance signals of the initial system, and also outputs of keys with large times. In the Mason algorithm, connections between these knots are determined and the intermediate count of 1 st level is formed.

Further, all white knots of the intermediate graph are replaced with black knots, with the index of variables of $T N$, which corresponds to discrete transformation of variables in time of $T N$, and the transmission functions of connections are replaced by their discrete transformations in time of $T N$. Black knots that are proper discrete variables for large times remain unchanged. The discrete count of the system of 1 st level is formed in the same manner. We set, where necessary, single connections for proper identical knots.

3. The component graph of 1 st level is formed. It turns out to be a combination of the initial graph of the system and discrete graph of 1st level. Thus the proper black knots of the graph, being weakened signals of keys $T i$, are united by single connections, $i=1, ., N$. A component graph of 1 st level is the basis for the construction of discrete graph of 2 nd level.

4. Algorithms for the discrete graph of 2nd level, et cetera till $N$-th number of levels 2 , are formed as per item a, with the change that in place of keys with time of $T N$, keys are utilized accordingly with time of $T N-1$ et cetera, till time of $T_{l}$. As a result of the expounded procedure, $N$ number of discrete counts of the system will be formed.

5. The final count of the system is further formed, which turns out to be a combination of the initial and $N$ of discrete counts of the system. By single connections, the accordance of knots of discrete counts is set with the entrance knots of the initial count.

6. In the algorithm of Mason, the input-output correlations are determined for the system variables of multirate continuously-discrete system with multiple times.

\section{Conclusion}

The offered method provides a formalised procedure of construction of input-output correlations of multirate continuously-discrete systems. The equivalent presentation of the mathematical model of multivariative multi-stage system makes it possible to provide the analysis for the one- time systems, and it simplifies greatly the whole process of research and allows developing the elements of computerized analysis systems and systems of designing multivariative multi-stage discrete-continuous control systems.

Observed multirate systems applications in the form of equivalent one-time models give common reasons for process analysis in given one-time systems. While observing resultant correlation, it is not difficult to see that in all cases impulse images of equivalent models outputs have the form of rational functions of given variables. Turning to Z-images we will have an equation defining the output images as of $\mathrm{z}$. They correspond to:

$$
Y(k T)=\frac{1}{2 \pi j} \oint f(z) z^{k-1} d z
$$

The closed contour of integration in equation (14) covers all poles $\mathrm{f}(\mathrm{z})$ [11]. Thus, the problem of process analysis in given multirate systems becomes reduced to the problem of rational functions of pole distribution analysis $\mathrm{f}(\mathrm{z})$ concerning a single circle.

\section{References}

[1] Araki M., Yamamoto K. (1986). Multivariable multirate sampled-data systems: statespace description, transfer characteristics, and Nyquist criterion, IEEE Trans. On Control, vol.31.

[2] Sauro Longhi (1994). Structural properties of multirate sampled-data systems, IEEE Transaction on Automatic Control, vol. 39, No. 3.

[3] Berg M.C., Amit N., Powell J.D. (1988). Multirate digital control system design, IEEE Trans. Auto. Contr., vol.AC33, No.12.

[4] Svetlichny, P., Kramar, V. (2009). Building of festo servo-motor imitation model using linear ARX-model, 2009 Annals of DAAAM and Proceedings of the International DAAAM Symposium

[5] Sekala A., Swider J. (2005). Hybrid graphs in modeling and analysis of discrete - continuous mechanical systems Proc. 13th International Scientific Conference on Achievements in Mechanical and Materials Engineering.

[6] Sedlar M. Bekey G. (1967). Signal Flow Graphs of Sampled-Data Systems: A New formulation IEEE Trans. on Automatic Control, AC-12, 2.

[7] Coffey T.C., Williams I.J. (1966). Stability Analysis of Multiloop, Multirate, Sampled Systems AIAA Journal, 4.

[8] Mason S.J. (1956). Feedback Theory - Further Properties of Signal Flow Graphs Proc. IRE, 41.

[9] Kuo B. (1986).Teorija i proektirovanie cifrovykh sistem upravlenija.-M.:Mashinostroenie.

[10] Kuo B.C. (1961). Composite Flow Graph Technique for the Solution of Multiloop, Multisampler, Sampled Systems IRE Trans. on Automatic Control, 1961.-Vol. AC-6, , pp.343-344

[11] Sain, M. K., Schrader C. B. (1990). The role of zeros in the performance of multi-input, multi-outputfeedback systems IEEE Transactions on Education №33. 